\title{
STALP: Style Transfer with Auxiliary Limited Pairing
}

\author{
D. Futschik ${ }^{1}$, M. Kučera ${ }^{1}$, M. Lukáč ${ }^{2}$, Z. Wang ${ }^{2}$, E. Shechtman ${ }^{2}$, D. Sýkora ${ }^{1}$ \\ ${ }^{1}$ Czech Technical University in Prague, Faculty of Electrical Engineering, Czech Republic \\ ${ }^{2}$ Adobe Research, USA
}

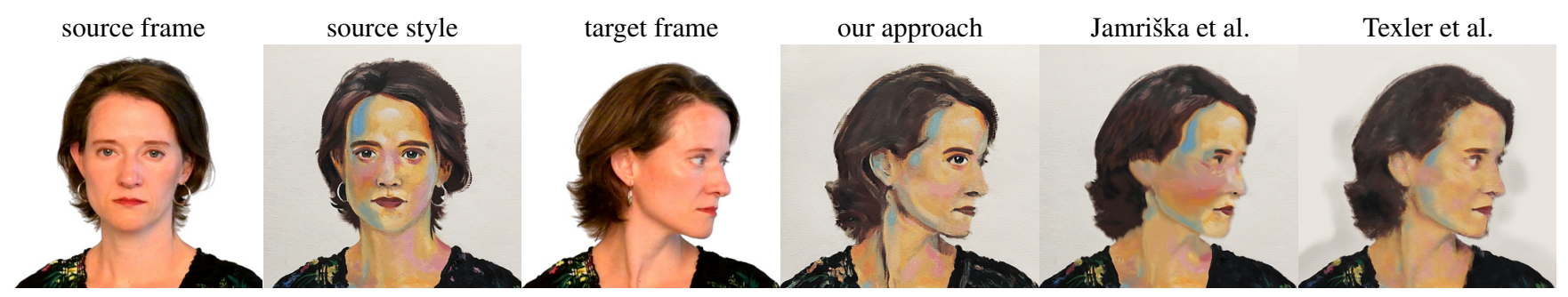

Figure 1: An example of style transfer with auxiliary pairing — an artist prepares a stylized version (source style) of a selected frame from input video (source frame). Then a network is trained to transfer artist's style to remaining video frames (target frame). During the training phase a subset of input video frames as well as the source frame and its stylized counterpart are taken into account. Once the network is trained, the entire sequence can be stylized in real-time (our approach). In contrast to current state-of-the-art in example-based video stylzation (Jamriška et al. [JST*19] and Texler et al. [TFK*20]) our approach better preserves important visual characteristics of the style exemplar even though the scene structure changed considerably (head rotation). Input video frames and source style @ Zuzana Studená, used with permission.

\begin{abstract}
We present an approach to example-based stylization of images that uses a single pair of a source image and its stylized counterpart. We demonstrate how to train an image translation network that can perform real-time semantically meaningful style transfer to a set of target images with similar content as the source image. A key added value of our approach is that it considers also consistency of target images during training. Although those have no stylized counterparts, we constrain the translation to keep the statistics of neural responses compatible with those extracted from the stylized source. In contrast to concurrent techniques that use a similar input, our approach better preserves important visual characteristics of the source style and can deliver temporally stable results without the need to explicitly handle temporal consistency. We demonstrate its practical utility on various applications including video stylization, style transfer to panoramas, faces, and 3D models.
\end{abstract}

\section{CCS Concepts}

- Computing methodologies $\rightarrow$ Non-photorealistic rendering;

\section{Introduction}

In recent years, methods for performing automatic style transfer from an exemplar image to a target image or a video have gained significant popularity. Although state of the art in this field progresses quickly and produces ever more believable artistic images, there are still aspects in which most methods tend to have fundamental shortcomings. One such crucial element is defining the semantic intent while still preserving visual characteristics of the used artistic media.

A seminal work in this direction was the Image Analogies frame- work introduced by Hertzmann et al. [HJO*01], which requires the user to provide a set of guidance channels $\left[\mathrm{BCK}^{*} 13, \mathrm{FJL}^{*} 16\right.$, FJS*17, JST* ${ }^{*}$ 19] that encourage the synthesis algorithm to transfer smaller patches of the style exemplar onto desired spatial locations in the target image. Those channels, however, need to be prepared explicitly by the user or generated algorithmically for a limited target domain, e.g., 3D renders [FJL*16], facial images [FJS*17], or a sequence of video frames close to the stylized keyframe [JST*19]. Deriving consistent semantically meaningful guidance in the general case remains an open problem. 
Neural approaches to style transfer [GEB16, LFY*17, KSS19] rely on the assumption that one can encode semantic similarity using the correspondence of statistics of neural features extracted from responses of the VGG network [SZ14]. Although such an assumption holds in some cases, it is not easy to amend when it fails. Moreover, in contrast to patch-based methods, neural techniques tend to produce noticeable visual artifacts due to their statistical nature. One can partially alleviate this drawback by applying patchbased synthesis in the neural domain [LW16, LYY*17]. However, since in this scenario neural features are transferred explicitly, the requirement of knowledge of accurate correspondences is still inevitable.

Another possibility of preserving semantically meaningful transfer is using the image-to-image translation principle pioneered by Isola et al. [IZZE17]. This approach can encode semantic intent and retain high-quality output. However, it has a fundamental limitation of requiring a relatively large dataset of image pairs (original image plus its stylized counterpart), which is rarely easy to obtain when considering artistic applications. Lastly, a group of unpaired image translation algorithms could be used [ZPIE17, PEZZ20], however, since it can be difficult to incorporate intent into these methods, they are not as suitable for tasks where the artist needs greater control.

In this paper, we present a novel approach to neural style transfer that allows artists to stylize a set of images with arbitrary yet similar content in a semantically meaningful way, while preserving the target subjects' critical structural features. In contrast to previous neural techniques, in our framework, the user explicitly encodes the semantic intent by specifying a stylized counterpart for a selected image from the set that needs to be stylized. Using this single style exemplar, we then train an image-to-image translation network that stylizes the remaining images. Our approach bears a resemblance to the recent keyframe-based video stylization framework of Texler et al. [TFK ${ }^{*} 20$ ], where a similar workflow is used. A key difference in our technique is that we consider other frames from the input sequence during the training phase. This enables us to ensure temporal stability without explicit guidance and better preserve style when the remaining video frames deviate from the original keyframe. Moreover, thanks to this increased robustness, our framework goes beyond video stylization. One can use it also in more challenging scenarios, including auto-completion of a panorama painting, stylization of $3 \mathrm{D}$ renders, or different portraits captured under similar illumination conditions.

\section{Related Work}

Despite the renewed interest and broader impact, image stylization algorithms date back decades. Traditionally, they were based on predefined, hand-designed transformations limited to a subset of styles, and possibly target domains as well. One example of such transformation approach was shown by Curtis et al. [CAS*97], running a physical simulation to produce watercolor filter effect. Other research directions focused on composing images from static or procedurally generated brush strokes or pens $\left[\mathrm{BLV}^{*} 10, \mathrm{BKTS} 06\right.$, PHWF01, SWHS97]. These conventional algorithmic approaches can create very appealing results, but they have the added difficulty of requiring the style filters to be designed on an individual basis.
Therefore, the act of creating a new style or even slight modifications of existing styles tends to necessitate considerable amounts of effort. These methods do not require a style exemplar, but instead contain a prior given by the design of the filter.

The framework of Image Analogies proposed by Hertzmann et al. [HJO*01] trades designing elements of the output image directly for designing a set of guidance channels which form a loss function. Optimizing over pixel locations and directly copying patches of an exemplar image guarantees that features found in the exemplar will be represented exactly in the resulting image. This framework became the basis of numerous style transfer

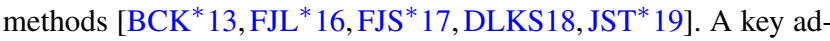
vantage over traditional algorithmic methods lies the fact that this framework allows for transfer of arbitrary style.

However, creating the guidance channels is cumbersome, and in some potential applications it might not be always clear how to design algorithms for obtaining them automatically, and still, the task of preparing a framework that would work with arbitrary images remains seemingly impossible. To sidestep this issue, methods of general style transfer have been formulated. Frigo et al. [FSDH16] attempts to re-imagine the problem of guiding channels by splitting the image into partitions and matching these to their counterparts. More commonly known, Gatys et al. [GEB16] uses responses of a neural network to generate global style statistics which an optimization process sees to reproduce in the result while incorporating a content constraint to prevent the overall structure from diverging too far from the target image. Refining these ideas to a video domain and employing a more sophisticated loss functions, others [CLY*17, $\left.\mathrm{LFY}^{*} 17, \mathrm{RDB} 18, \mathrm{KSS} 19\right]$ manage to produce results which are coherent in time and more faithful to the style. While they produce impressive results on some inputs, these methods generally take all the control out of the artists' hands and are notoriously difficult to steer in different directions, as their mechanisms are non-intuitive and unpredictable.

A different view of the problem is offered by the image-to-image framework, which aims to translate images from one domain to another, which is directly applicable to style transfer. While the original image translation methods [IZZE17,JAFF16] require relatively large dataset to work reliably, by their combination with generative adversarial models [GPAM*14, ZPIE17], this requirement can be relaxed. Unlike techniques based on image analogies, these methods tend to require substantial amount of model training. And although patch-based synthesis [FJS*17] can be used to generate a large number of image pairs on which one can train the image-toimage translation network [FCC $\left.{ }^{*} 19\right]$, the problem of having meaningful guidance remains.

Few-shot learning techniques [LHM*19, WLT*19], as well as approaches based on deformation transfer [SLT*19b, SLT*19a] require only a single style exemplar. However, they still need pretraining on large dataset of specific target domains and thus are not applicable in general case. Moreover, these techniques capture only the target subject's coarse deformation characteristics; its structure or identity is omitted. A similar drawback also holds for approaches based on generative adversarial networks such as StyleGAN v2 [KLA*20]. In this approach, a massive collection of artworks is used to train a network that can generate an artistic image 
for a given input latent vector. Those vectors can then be predicted and fine-tuned to align the generated image with the target image's features. However, this process is inaccurate, leading to imprecise alignment that hinders the network's ability to preserve the target subject's structure or identity.

\section{Our Approach}

As input to our method, we take pairs of images $K=(X, Y)$ called keyframes. They represent a visual translation from a source visual domain of $X$ into a target domain of $Y$. For instance $X$ can be a photo and $Y$ its stylized counterpart prepared by an artist (see Fig. 2). Note that our key assumption about $K$ is that it should be as small as possible, in practice even a single keyframe is usually sufficient. This is in line with our central motivation to reduce the amount of manual work since the creation of keyframes is time consuming and thus prohibitive. In addition to $K$, the user also provides a set of unpaired images $Z$, which they would like to stylize. The images in $Z$ can be arbitrary, but our method works best if their domain is similar or same as $X$. For instance $Z$ and $X$ can be frames from the same video sequence or photos from the same location, etc. If there is a larger number of images in $Z$, it is beneficial to prune it as smaller number of images in $Z$ usually has a positive effect on the resulting quality (see Fig. 8). Both keyframes $K$ as well as unpaired images $Z$ are used during an optimization process that produces a neural translation model $\mathcal{F}$. Using $\mathcal{F}$ one can stylize $Z$ in a semantically meaningful way, i.e., produce a set of output images $O$ in which important visual features of artistic style $Y$ are reproduced at appropriate locations.

As $\mathcal{F}$, we use the network architecture design of Futschik et al. [FCC ${ }^{*}$ 19] (see Fig. 3), a U-Net-type network, which is particularly suitable for style transfer tasks as it allows to reproduce important high-frequency details that are crucial for generating believable artistic styles. In the original method of Futschik et al. $\mathcal{F}$ was trained on a large dataset of $K$ which is intractable in our scenario. Texler et al. [TFK ${ }^{*} 20$ ] uses the network architecture of $\mathcal{F}$ as well in a similar setting as ours, i.e., small number of keyframes $K$, however, their method struggles with larger structural changes in the target images $Z$.

To address this issue, we leverage the fact that the set of target images $Z$ is known beforehand and thus we can incorporate this additional knowledge into the optimization process. To do that, we introduce a different training strategy. The process is a combination of two complementary objectives, illustrated in Fig. 2, which we minimize as we train $\mathcal{F}$ :

- $L_{1}$ loss on the original translation pairs $K$, ensuring that keyframes are represented as closely as possible.

- VGG loss between the images from set $Z$ and set $Y$, which acts as a regularizer for the stylized images $O$.

Combining these two, we obtain the objective function we would like to minimize:

$$
\sum_{i}\left|\mathcal{F}\left(X_{i} ; \theta\right)-Y_{i}\right|+\lambda \sum_{j, k} \sum_{l}\left\|\mathcal{G}^{l}\left(\mathcal{F}\left(Z_{j} ; \theta\right)\right)-\mathcal{G}^{l}\left(Y_{k}\right)\right\|^{2}
$$

where $\theta$ is a set of weights of $\mathcal{F}$ which we would like to optimize, $\mathcal{G}^{l}$ stands for Gram correlation matrix calculated at layer $l \in L$ after extracting VGG network responses [SZ14] of the given image, and $\lambda$ is a weighting coefficient which we set to $100 /(|Z||L|)$ for all conducted experiments.

Contrary to previous techniques [GEB16, JAFF16] which compute Gram matrix from a subset of layers we found that evaluating the loss at every layer $l \in L$ of VGG is beneficial in terms of measuring the overall style quality. However, this is computationally more expensive and thus our method generally requires an order of magnitude more time to produce the final results. These previous methods use the term purely as a proxy for style transfer. In our case we use it as regularizer to prevent the model from overfitting to the keyframes. This effect is visible in Fig. 4, where if we take away the VGG loss, the resulting $\mathcal{F}$ is unable to generalize beyond $K$ whereas using VGG loss only will negatively affect the content.
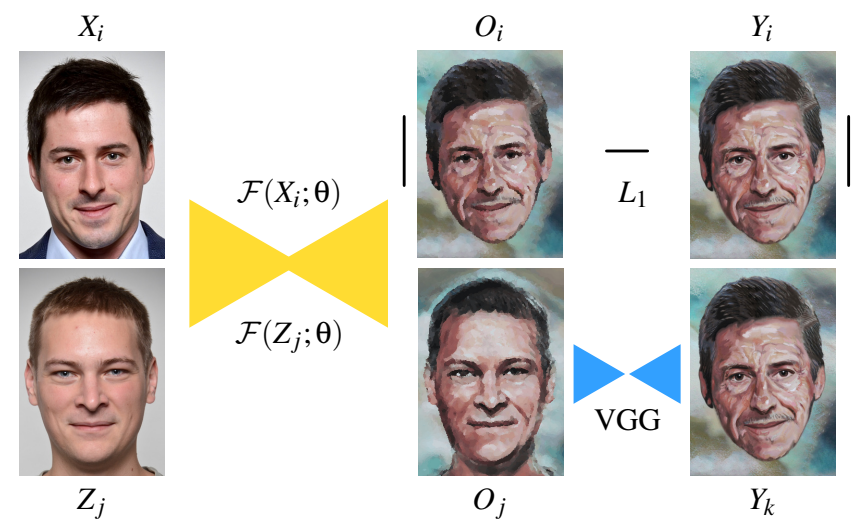

Figure 2: An overview of our approach-we optimize weights $\theta$ of a translation network $\mathcal{F}$ which accepts images from a source domain $X$ or $Z$ and produces output images $O$ with a similar appearance as those in the target domain $Y$. The high-frequency details are preserved well, thanks to the $L_{1}$ loss computed on the artistcreated style images $Y$ which have the same structure as the input images $X$, while the style consistency on other images $Z$ is enforced due to the VGG loss. Source style (C) Graciela Bombalova-Bogra, used with permission.

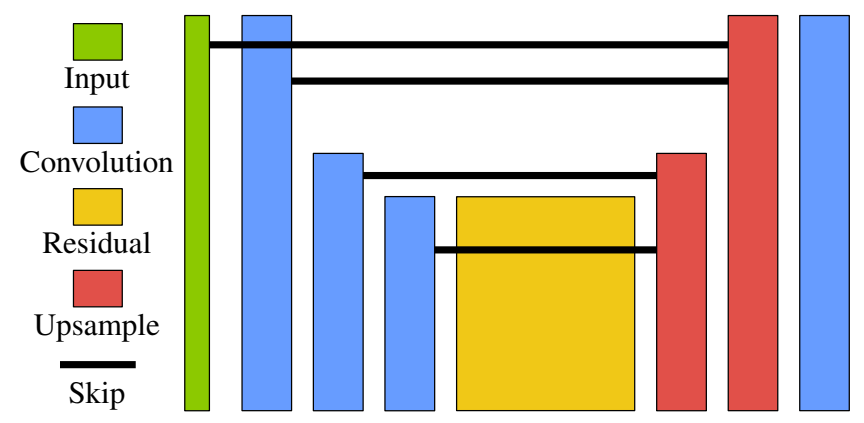

Figure 3: A network architecture used for our model $\mathcal{F}$ : input layer (green), one $7 \times 7$ and two $3 \times 3$ convolution blocks (blue), nine $3 \times 3$ residual blocks (yellow), two $3 \times 3$ upsampling blocks (red), and one additional block with $7 \times 7$ convolutions (blue). Skip connections (black) are used to connect downsampling and upsampling layers. 

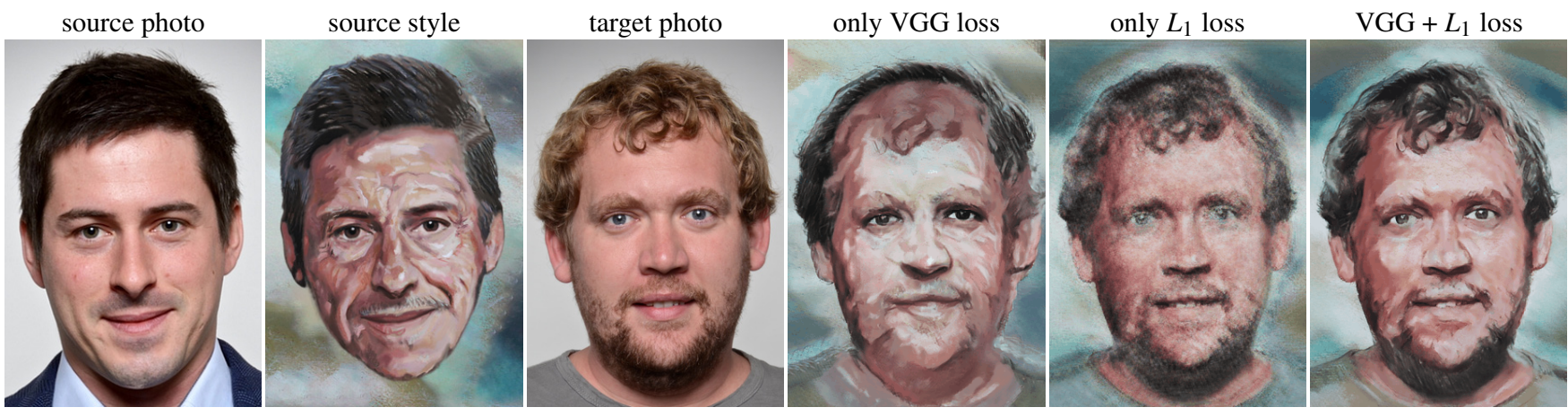

Figure 4: An ablation study demonstrating the importance of individual terms in our objective function (1)—a stylized pair ( $\left.X_{1}, Y_{1}\right)($ source photo, source style) is used together with $Z_{1}$ (target photo) to optimize weights of model $\mathcal{F}$. When only VGG loss is used, the identity of a person in the target photo deteriorates. On the other hand when only $L_{1}$ loss is used during optimization source, style is not preserved well. By combining $L_{1}$ loss and VGG loss in (1) we get the result which produces a good balance between identity and style preservation. Source style (C) Graciela Bombalova-Bogra, used with permission.

By minimizing the objective (1) we produce a trained model $\mathcal{F}$, which in turn is able to stylize the images from $Z$ via a feedforward pass. An important aspect to notice is that unlike most previous style transfer techniques, our approach does not enforce any content loss explicitly. We find that content losses found in literature [GEB16, KSS19] tend to be detrimental to the quality of style transfer, especially when higher frequencies are concerned. It causes a particular washed-out look where important style details are missing (see Fig. 5). An objection to our argument could be that without explicit penalty on the content preservation, the model can resort to memorizing the keyframes and return $Y$ regardless the content in target images $Z$. This would eventually minimize both the $L_{1}$ error as well as the VGG loss. The reason why the optimization process does not end up using this trivial solution is twofold. We argue that due to the limited receptive field of $\mathcal{F}$, it has to learn an effective encoding of the input; in addition, since the VGG loss is relatively weak and serves only as a non-linear regularizer, it makes the trivial solution difficult to find during the optimization process. Moreover, by optimizing a one-to-one mapping between images of perceptually similar semantic structure $(X$ to $Y$ ), we posit that this acts as an implicit content preservation technique.

\section{Results}

We implemented our approach using PyTorch [PGM*19]. For all experiments, we use Adam optimizer with learning rate $10^{-4}$, $\beta_{1}=0.9, \beta_{2}=0.999$. We found that higher rate does not work well when performing many Gram matrix operations that are prone to producing exploding gradients. For the network model $\mathcal{F}$, we use 9 residual blocks, which is in line with previous approaches $\left[\mathrm{FCC}^{*} 19, \mathrm{TFK}^{*} 20\right]$. However, since in our optimization batch size is equal to 1 we use instance normalization [UVL16] instead of batch normalization. All layers used for Gram matrix computation are post-activated with ReLU to better incorporate nonlinearity. In each experiment, we let the optimization process run for approximately 100k iterations, which translates into roughly 36 hours of wall time on a single NVIDIA V100 GPU, depending on the target resolution. The resolutions we produce range from

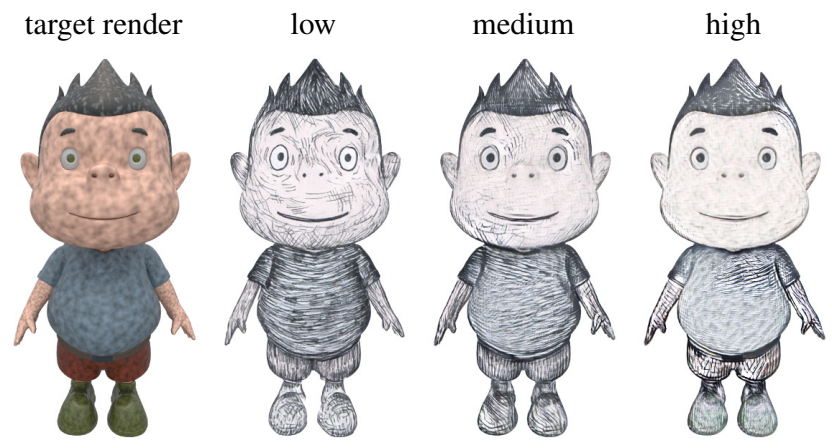

Figure 5: An illustration of a wash-out effect caused by adding an explicit content loss term [KSS19] into our objective function (1). Target render stylized using model $\mathcal{F}$ optimized on a stylized pair from Fig. 9 with low, medium, and high content loss weight. Note how style details deteriorate gradually with the increasing content loss. Source style @ Štépánka Sýkorová, used with permission.

512 px to 768 px as longer side of the image, with the shorter side scaled appropriately to preserve correct aspect ratio given by the input images.

We evaluated our approach in five different use cases to demonstrate its wider range of applicability: (1) keyframe-based video stylization, (2) style transfer to 3D models, (3) autopainting panorama images, (4) example-based stylization of portraits, and (5) real-time stylization of video calls.

Video stylization results together with a side-by-side comparison of the output from previous techniques [JST*19, TFK ${ }^{*} 20$ ] is presented in Figures 1 and 6 as well as in our supplementary video. In each experiment, we selected a keyframe $X$ from the input video sequence $V$ which was stylized by an artist to produce $Y$. Then a $10 \%$ of video frames from $V$ were sampled uniformly to get the set $Z$. Using this input, the weights $\theta$ of the network $\mathcal{F}$ were optimized and used to stylize the entire sequence $V$. In Fig. 7 we compare the scenario where multiple keyframes $K$ are used to stylize $V$. We also 

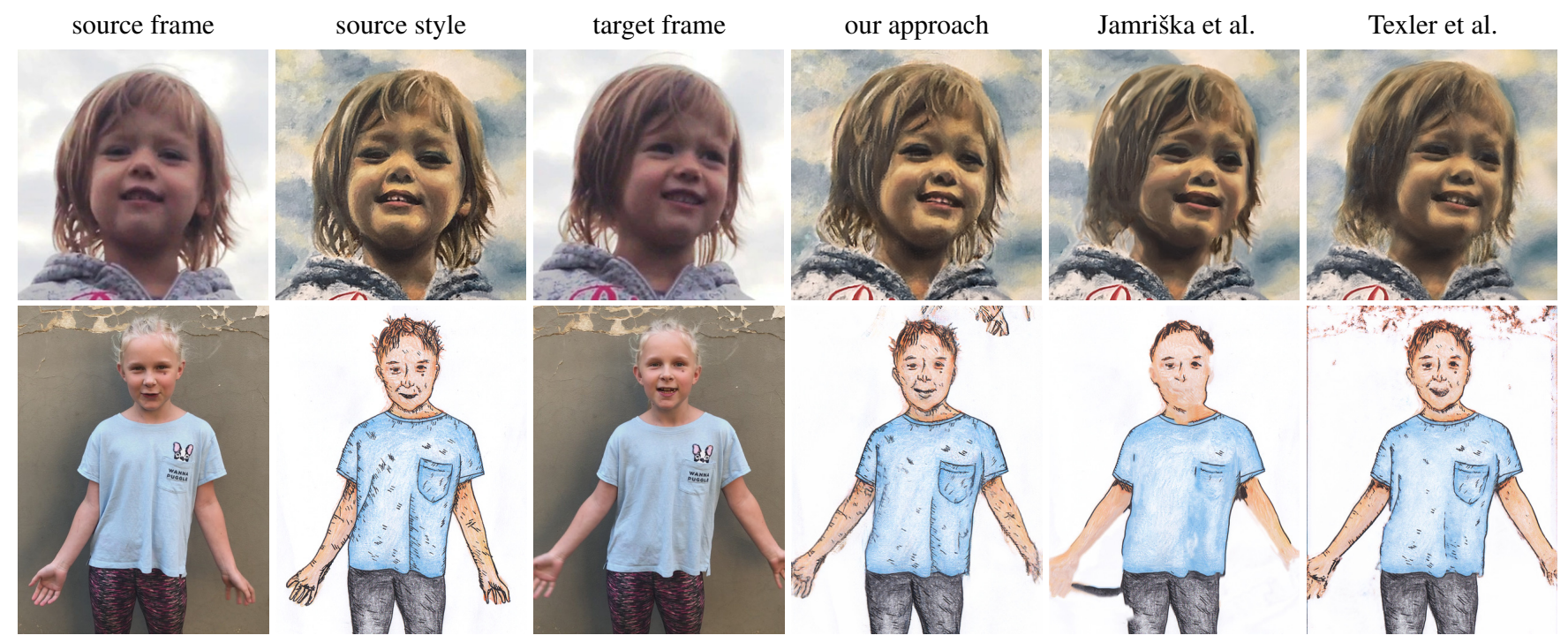

Figure 6: Video stylization results—in each video sequence (rows) a selected frame (source frame) is stylized using different artistic media (source style). The network is then trained using this stylized pair and a subset of frames from the entire video sequence (target frame). The results of our method (our approach) are compared with the output of concurrent techniques: Jamriška et al. [JST*19] and Texler et al. [TFK ${ }^{*}$ 20]. Note how our method better preserves important style details and visual features of the target frames. Previous style transfer techniques tend to produce wash out artifacts due to significant structural changes with respect to the source frame. Video frames and style (top row) () Zuzana Studená, and (bottom row) @ Štěpánka Sýkorová, used with permission.

considered an option that all frames from $V$ are used as $Z$, or instead of using uniform sampling we selected $10 \%$ of frames that represent the most signficant changes in the scene. We found that sparse uniform sampling has usually the best performace (see Fig. 8).

As visible from the results and comparisons, our approach can better preserve style details during a longer time frame even if the scene structure changes considerably with respect to $X$. Also, note how the resulting stylized sequence has better temporal stability implicitly without performing any additional treatment, which contrasts with previous techniques [JST*19, TFK ${ }^{*} 20$ ] that need to handle temporal consistency explicitly.

Style transfer to 3D models resembles video stylization use case, however, there are specific features worth separate discussion. In this scenario we let the user select a camera viewpoint from which a 3D model is rendered to get image $X$. As the network $\mathcal{F}$ is sensitive to local variations in $X$, it is important to avoid larger flat regions which can make the translation ambiguous. Due to this reason we add a noisy texture to the 3D model to alleviate the ambiguity (see source render in Fig. 9). An artist then prepares the stylized counterpart $Y$ and the model is rendered again from a few different viewpoints to produce $Z$. Using those inputs, weights $\theta$ of the network $\mathcal{F}$ are optimized and the translation network can then be used in an interactive scenario where the user changes the camera viewpoint, the 3D model is rendered on the fly, and immediately stylized using $\mathcal{F}$. See Figures 9 and 10 and our supplementary video for results in this scenario. As in the video stylization case when compared to other techniques [GEB16, KSS19, JST*19, TFK*20] our approach better preserves the style exemplar (c.f. Fig. 9) and implicitly maintains temporal consistency.
In the panorama auto-painting scenario we consider a set of photos $P$ taken from the same location by rotating the camera around its center of projection. We compute a set of homographies $H$ between photos in $P$ using the method of Brown et al. [BL07]. Then we let the artist pick one photo from $P$ as $X$ and produce its stylized counterpart $Y$. Remaining photos in $P$ are used as $Z$. After the optimization one can use $\mathcal{F}$ to stylize all photos in $P$, stitch them together using $H$, and either produce a cylindrical unwrap or alternatively use an interactive scenario where the user changes the relative camera rotation from which a pinhole projection can be computed and stylized in real-time using $\mathcal{F}$. As visible in Fig. 11 and 12 from the comparisons with [LYY* 17, KSS19] our approach better preserves the original style details as well as semantic context.

In the example-based portrait stylization use case a set of portraits $U$ is assumed to be taken under similar lighting conditions. One portrait from $U$ is used as $X$ and stylized to get $Y$. The rest of portraits in $U$ is used in $Z$. Resulting model $\mathcal{F}$ can then be used to stylize all portraits in $U$. In Fig. 13 stylization results for two different style exemplars are presented. It is apparent that our approach produces a reasonable compromise between identity and style preservation whereas previous neural methods such as [GEB16, KSS19] tend to preserve identity better, but lose style details. On the other hand, patch-based technique [FJS*17] reproduces style better, nevertheless, has difficulties retaining identity.

In real-time stylization of video calls we let the user record a short video sequence $V$ which captures her face during a regular video meet. A most representative frame is selected from $V$ and used as $X$. An artist then produces its stylized counterpart $Y$ 


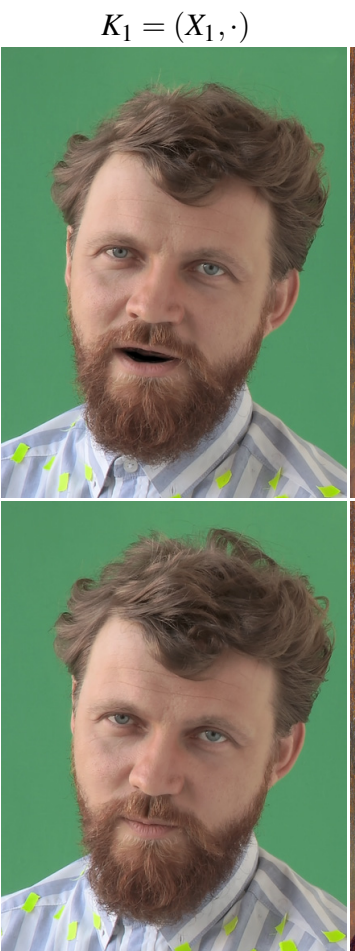

$K_{2}=\left(X_{2}, \cdot\right)$
$K_{1}=\left(\cdot, Y_{1}\right)$
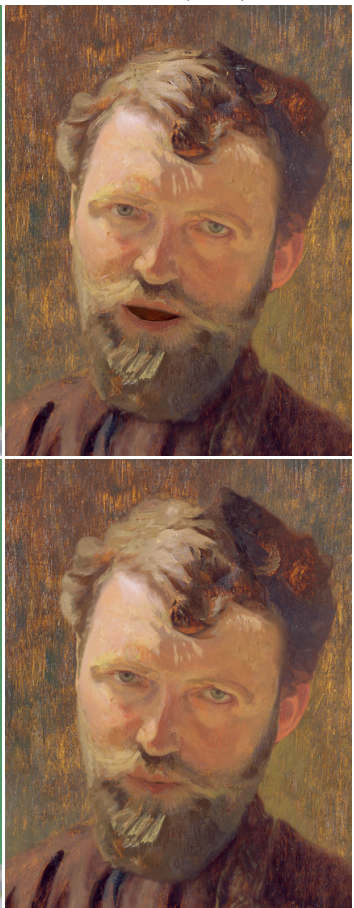

$K_{2}=\left(\cdot, Y_{2}\right)$
$Z_{1}$
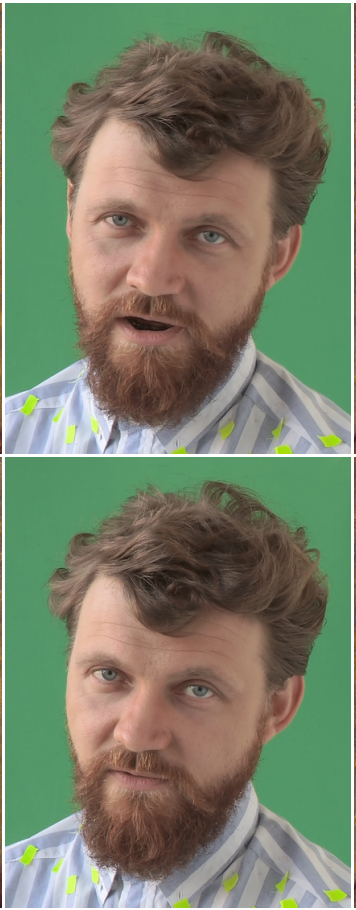

$Z_{2}$
$O_{1}$ (with $K_{1}$ )
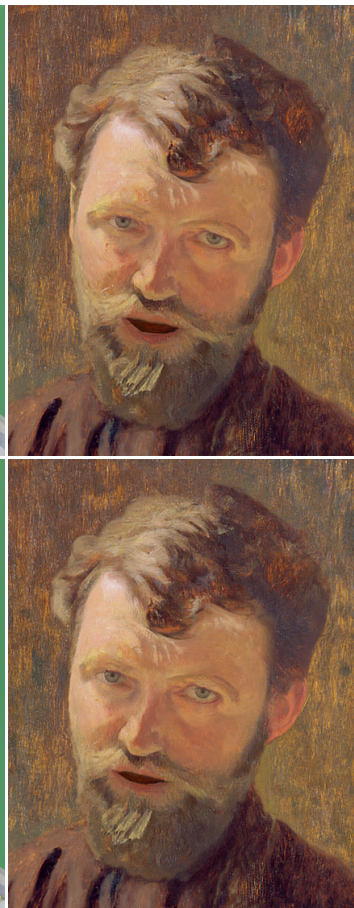

$\mathrm{O}_{2}$ (with $\left.K_{1}\right)$
$O_{1}\left(\right.$ with $\left.K_{1} \& K_{2}\right)$

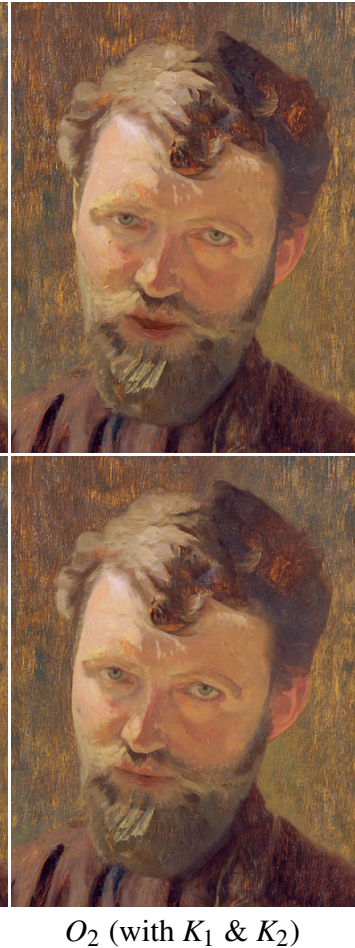

Figure 7: Example of video stylization with multiple keyframes-two keyframes $K_{1}=\left(X_{1}, Y_{1}\right)$ and $K_{2}=\left(X_{2}, Y_{2}\right)$ were created by painting over the input video frames $X_{1} \& X_{2}$ to get their stylized counterparts $Y_{1} \& Y_{2}$. First, our network $\mathcal{F}$ was trained using only single keyframe $K_{1}$ and applied to stylize input video frames $Z_{1} \& Z_{2}$ to produce $O_{1} \& O_{2}$ (with $K_{1}$ ). Note, how closed mouth in $Z_{2}$ was not stylized properly in $\mathrm{O}_{2}$ (with $K_{1}$ ). By adding $K_{2}$ to the list of keyframes used during training phase, open and closed mouth is stylized better, see $O_{1} \& O_{2}$ (with $K_{1} \& K_{2}$ ). Frames $X_{1}, X_{2}, Y_{1}, Y_{2}, Z_{1} \& Z_{2} \odot$ Muchalogy, used with permission.

and $10 \%$ of other frames in $V$ are used as $Z$. A model $\mathcal{F}$ is optimized using those inputs. Then, during the next video call $\mathcal{F}$ is used to stylize captured video frames in real-time. See Fig. 14 and our supplementary video for an example of such interactive stylized video call. From the comparison with the method of Texler et al. $\left[\mathrm{TFK}^{*} 20\right]$ it is visible that our approach not only better preserves the overall style quality but also retains temporal stability which is difficult to accomplish by the method of Texler et al. in this kind of interactive scenario.

\subsection{Perceptual study}

In order to qualitatively evaluate our approach, we performed a perception study comparing the outputs of our method with the outputs of three state-of-the-art techniques (Jamriška et al. [JST*19], Kolkin et al. [KSS19], and Texler et al. [TFF*20] (green points)). In our experiment we wanted to evaluate how well our method reproduces the given artistic style and how well it preserves the content of the target image. To perform the evaluation, we collected data via an online survey, where we presented 170 participants with a randomized set of comparisons (2AFC) asking to choose which anonymized stylization reproduces style or preserves content better. In total each participant responded to 28 questions. In each ques- tion, an output from a different method was paired with the output from our technique using the same input data.

The measured preference scores of our method compared to other techniques can be seen in Fig. 15. We set out a null hypothesis that "there is no statistically significant difference in the content preservation or style reproduction between the results of our method and the other methods." Then we discussed the probability of rejection of the null hypothesis using the data we collected via Student's t-test. In the style reproduction category, we were able to reject the null hypothesis with more then $99 \%$ probability in comparison to all tested methods in favor of our method. In the content preservation category, we were able to reject the null hypothesis with more than $99 \%$ probability, but only the comparison with the method of Jamriška et al. was in favor of our method while the other two were not.

\section{Limitations and Future Work}

While our approach improves on current state-of-the-art in example-based stylization, we have observed some limitations in how it can be applied.

The most important limitation as compared to related approaches 


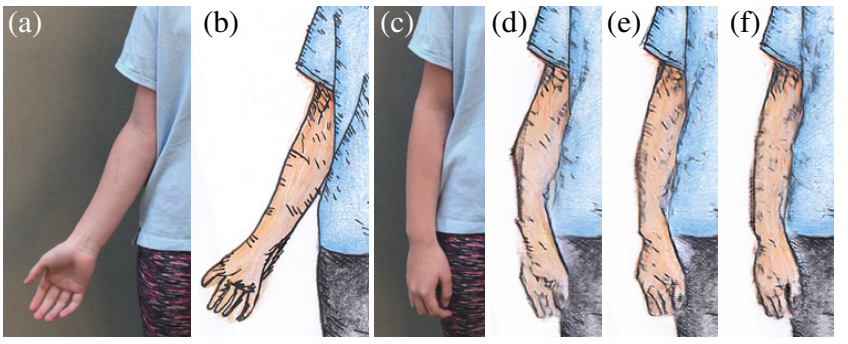

Figure 8: A different sampling strategy for a selection of frames in $Z$ - a source frame from a sequence $V(a)$ and its stylized counterpart $(b)$ are used as $K$. Then weights of $\mathcal{F}$ are optimized with $K$ and $Z$, where $Z$ contains all frames from $V(d), 10 \%$ of uniformly sampled frames from $V(e)$, and $10 \%$ of adaptively sampled frames from $V(f)$. Note how dense sampling tends to produce distortion artifacts on a rare hand pose (c) due to overfitting on a different pose that is more frequent in the sequence $V(a)$ whereas sparse sampling generalizes better. Source video frames $(a, c)$ and style $(b)$ (C) Štěpánka Sýkorová, used with permission.

is notably longer time frame required to finish the optimization, which might be prohibitive for artist's exploration. To alleviate this drawback we envision a combination of fast patch-based training strategy of Texler et al. [TFK*20] with the computation of VGG loss which needs to be performed in a full-frame setting.

Due to the usage of relatively computationally expensive neural network model, the maximum resolution is limited. While we are able to generate output images with resolutions greater than method of Texler et al. (e.g. $768 \times 768$ vs. $512 \times 512$ ), it is still significantly lower than what patch-based methods [JST*19] are capable of. As a future work we envision to alleviate this drawback by combining our neural approach with patch-based technique of [TFF* 20].

In our proposed workflow an artist is responsible for keyframe selection. While some rules of thumb can be applied, such as selecting a frame that contains all features that are descriptive for most other frames, a mechanism which would select the keyframe automatically would improve ease of use.

A key advantage of our approach over current state-of-the-art in example-based video stylization [JST ${ }^{*} 19, \mathrm{TFK}^{*} 20$ ] is greater robustness to structural discrepancies in the target frames. Even a relatively significant change such as head rotation is handled relatively well (see Fig. 1). In this case the network can successfully reproduce newly appearing content while still being able to preserve the notion of important planar structures of the original artistic media. On the other hand, some specific localized features such as eyes, may remain unchanged (see Fig. 16). A similar issue is known from visual attribute transfer approaches such as Deep Image Analogy [LYY* 17]. As compared to them our method is able to adopt to structural changes better (see Fig. 17).

Most significantly, the method does not seem to generalise very well for completely generic use cases, for example in Fig. 18, where input images are sampled from different underlying distributions. Thus the set of potential applications is limited to groups of images of visually similar settings created under comparable conditions.

\section{Conclusion}

We presented an approach of semantically meaningful style transfer that can leverage a limited number of paired exemplars to stylize a broader set of target images having similar content to the examples. We optimize weights of an existing image-to-image translation network by minimizing a novel kind of objective function that considers the consistency among the provided stylized pairs as well as discrepancy between VGG features of style exemplars and a subset of stylized target images.

Thanks to this combination, our approach can better preserve style details even when the target images' content differs significantly from the style exemplar. Moreover, our method implicitly maintains temporal consistency in the video stylization scenario, which needs to be treated explicitly in previous techniques. We demonstrated the benefits of our approach in numerous practical use cases, including style transfer to videos and faces, auto-painting of panorama images, and real-time stylization of 3D models and video calls.

\section{Acknowledgements}

We thank the anonymous reviewers for their valuable feedback and insightful comments. We are also grateful to Zuzana Studená, Štěpánka Sýkorová, Jolana Sýkorová, Graciela Bombalova-Bogra, Adrian Morgan, and Muchalogy for providing style exemplars and input video sequences. This research was supported by Adobe, the Grant Agency of the Czech Technical University in Prague, grant No. SGS19/179/OHK3/3T/13 (Research of Modern Computer Graphics Methods), and by the Research Center for Informatics, grant No. CZ.02.1.01/0.0/0.0/16_019/0000765.

\section{References}

[BCK*13] Bénard P., Cole F., Kass M., Mordatch I., Hegarty J., Senn M. S., Fleischer K., Pesare D., Breeden K.: Stylizing animation by example. ACM Transactions on Graphics 32, 4 (2013), 119.

[BKTS06] Bousseau A., Kaplan M., Thollot J., Sillion F. X.: Interactive watercolor rendering with temporal coherence and abstraction. In Proceedings of International Symposium on Non-Photorealistic Animation and Rendering (2006), pp. 141-149.

[BL07] BRown M., Lowe D. G.: Automatic panoramic image stitching using invariant features. International Journal of Computer Vision 74, 1 (2007), 59-73.

[BLV*10] Bénard P., Lagae A., Vangorp P., LefebVRe S., DretTAKIS G., THOLLOT J.: A dynamic noise primitive for coherent stylization. Computer Graphics Forum 29, 4 (2010), 1497-1506.

[CAS*97] Curtis C. J., Anderson S. E., Seims J. E., Fleischer K. W., SALESIN D. H.: Computer-generated watercolor. In SIGGRAPH Conference Proceedings (1997), pp. 421-430.

[CLY*17] Chen D., Liao J., Yuan L., Yu N., Hua G.: Coherent online video style transfer. In Proceedings of IEEE International Conference on Computer Vision (2017), pp. 1114-1123.

[DLKS18] DVORožŇÁK M., LI W., KiM V. G., SÝKora D.: ToonSynth: Example-based synthesis of hand-colored cartoon animations. ACM Transactions on Graphics 37, 4 (2018), 167.

[FCC*19] Futschik D., Chai M., Cao C., Ma C., Stoliar A., KoROLEV S., TUlYAKOV S., KUČERA M., SÝKORA D.: Real-time patchbased stylization of portraits using generative adversarial network. In Proceedings of the ACM/EG Expressive Symposium (2019), pp. 33-42. 


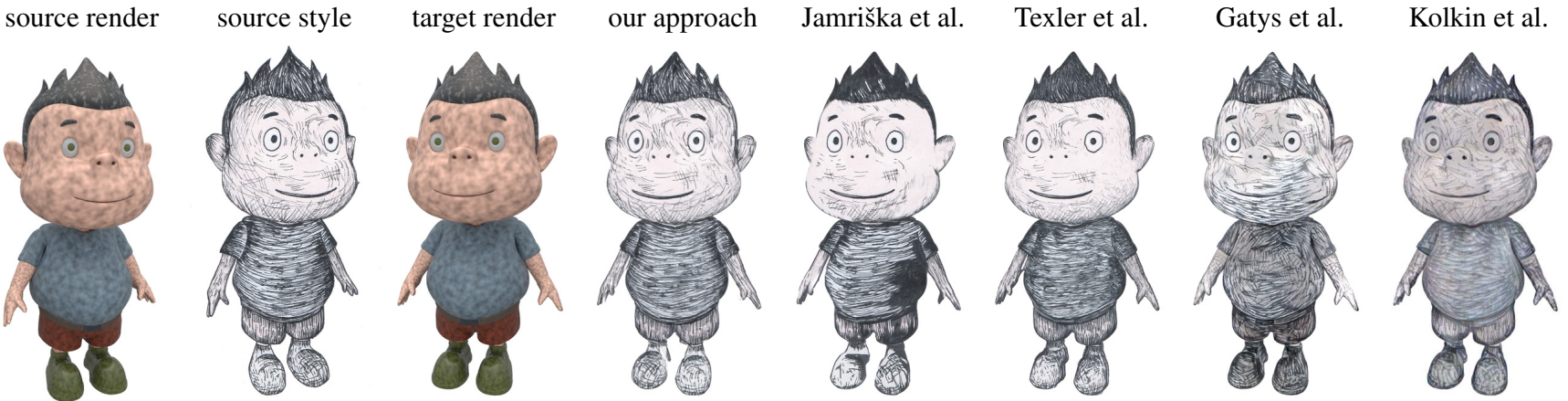

Figure 9: Stylization of 3D renders-a colored 3D model enhanced with an artificial noisy texture to avoid large flat regions (source render) is stylized at a selected viewpoint by an artist (source style). The network is then trained using the stylized pair and a set of additional renders of the same model viewed from a different direction (target render). The trained network can then be used to stylize the rendered $3 D$ model from a different user-specified position in real-time (our approach). When compared to other concurrent style transfer techniques (Jamriška et al. [JST*19], Texler et al. [TFK*20], Gatys et al. [GEB16], and Kolkin et al. [KSS19]) our approach better preserves important high-frequency details of the original style exemplar while being able to adopt to a new pose in a semantically meaningful way. Source style @ Štěpánka Sýkorová, used with permission.

[FJL*16] FiŠER J., JAMrišKa O., LuKÁČ M., Shechtman E., ASENTE P., LU J., SÝKORA D.: StyLit: Illumination-guided examplebased stylization of 3D renderings. ACM Transactions on Graphics 35, 4 (2016), 92.

[FJS*17] Fišer J., JamrišKa O., Simons D., Shechtman E., Lu J., ASENTE P., LUKÁČ M., SÝKORA D.: Example-based synthesis of stylized facial animations. ACM Transactions on Graphics 36, 4 (2017), 155.

[FSDH16] Frigo O., Sabater N., Delon J., Hellier P.: Split and match: Example-based adaptive patch sampling for unsupervised style transfer. In Proceedings of IEEE Conference on Computer Vision and Pattern Recognition (2016), pp. 553-561.

[GEB16] Gatys L. A., Ecker A. S., Bethge M.: Image style transfer using convolutional neural networks. In Proceedings of IEEE Conference on Computer Vision and Pattern Recognition (2016), pp. 24142423.

[GPAM*14] Goodfellow I. J., Pouget-Abadie J., Mirza M., Xu B., Warde-Farley D., Ozair S., Courville A. C., Bengio Y.: Generative adversarial nets. In Advances in Neural Information Processing Systems (2014), pp. 2672-2680.

[HJO*01] Hertzmann A., Jacobs C. E., Oliver N., Curless B., SALESIN D. H.: Image analogies. In SIGGRAPH Conference Proceedings (2001), pp. 327-340.

[IZZE17] Isola P., Zhu J.-Y., Zhou T., Efros A. A.: Image-toimage translation with conditional adversarial networks. pp. 5967-5976.

[JAFF16] Johnson J., Alahi A., Fei-Fei L.: Perceptual losses for real-time style transfer and super-resolution. In Proceedings of European Conference on Computer Vision (2016), pp. 694-711.

[JST*19] JamrišKa O., Sochorová S., TeXler O., Lukáč M., Fišer J., Lu J., Shechtman E., SÝkORA D.: Stylizing video by example. ACM Transactions on Graphics 38, 4 (2019), 107.

[KLA19] Karras T., LAine S., Aila T.: A style-based generator architecture for generative adversarial networks. In Proceedings of IEEE Conference on Computer Vision and Pattern Recognition (2019), pp. 4401-4410.

[KLA*20] KarRas T., Laine S., AitTala M., Hellsten J., LehtiNEN J., AILA T.: Analyzing and improving the image quality of stylegan. In Proceedings of IEEE Conference on Computer Vision and Pattern Recognition (2020), pp. 8107-8116.
[KSS19] Kolkin N. I., Salavon J., Shakhnarovich G.: Style transfer by relaxed optimal transport and self-similarity. In Proceedings of IEEE Conference on Computer Vision and Pattern Recognition (2019), pp. 10051-10060.

[LFY*17] Li Y., FAng C., Yang J., Wang Z., Lu X., Yang M.-H.: Universal style transfer via feature transforms. In Advances in Neural Information Processing Systems (2017), pp. 385-395.

[LHM*19] LiU M.-Y., Huang X., Mallya A., Karras T., Aila T., LEHTINEN J., KAUTZ J.: Few-shot unsupervised image-to-image translation. In Proceedings of IEEE International Conference on Computer Vision (2019), pp. 10551-10560.

[LW16] LI C., WAND M.: Combining markov random fields and convolutional neural networks for image synthesis. In Proceedings of IEEE Conference on Computer Vision and Pattern Recognition (2016), pp. 2479-2486.

[LYY*17] Liao J., Yao Y., Yuan L., Hua G., Kang S. B.: Visual attribute transfer through deep image analogy. ACM Transactions on Graphics 36, 4 (2017), 120.

[PEZZ20] PARK T., Efros A. A., Zhang R., ZhU J.-Y.: Contrastive learning for unpaired image-to-image translation. In European Conference on Computer Vision (2020).

[PGM*19] Paszke A., Gross S., Massa F., Lerer A., Bradbury J., Chanan G., Killeen T., Lin Z., Gimelshein N., Antiga L., Desmaison A., Kopf A., Yang E., DeVito Z., Raison M., TeJani A., Chilamkurthy S., Steiner B., Fang L., Bai J., ChinTALA S.: Pytorch: An imperative style, high-performance deep learning library. In Advances in Neural Information Processing Systems. 2019, pp. 8024-8035.

[PHWF01] Praun E., Hoppe H., Webb M., Finkelstein A.: Realtime hatching. In SIGGRAPH (2001), pp. 581-586.

[RDB18] RUDER M., DosovitskiY A., BRoX T.: Artistic style transfer for videos and spherical images. International Journal of Computer Vision 126, 11 (2018), 1199-1219.

[SLT*19a] Siarohin A., Lathuilière S., Tulyakov S., Ricci E., SEBE N.: Animating arbitrary objects via deep motion transfer. In Proceedings of IEEE Conference on Computer Vision and Pattern Recognition (2019), pp. 2377-2386.

[SLT*19b] Siarohin A., Lathuilière S., Tulyakov S., Ricci E., SEBE N.: First order motion model for image animation. In Advances in Neural Information Processing Systems (2019), pp. 7135-7145. 


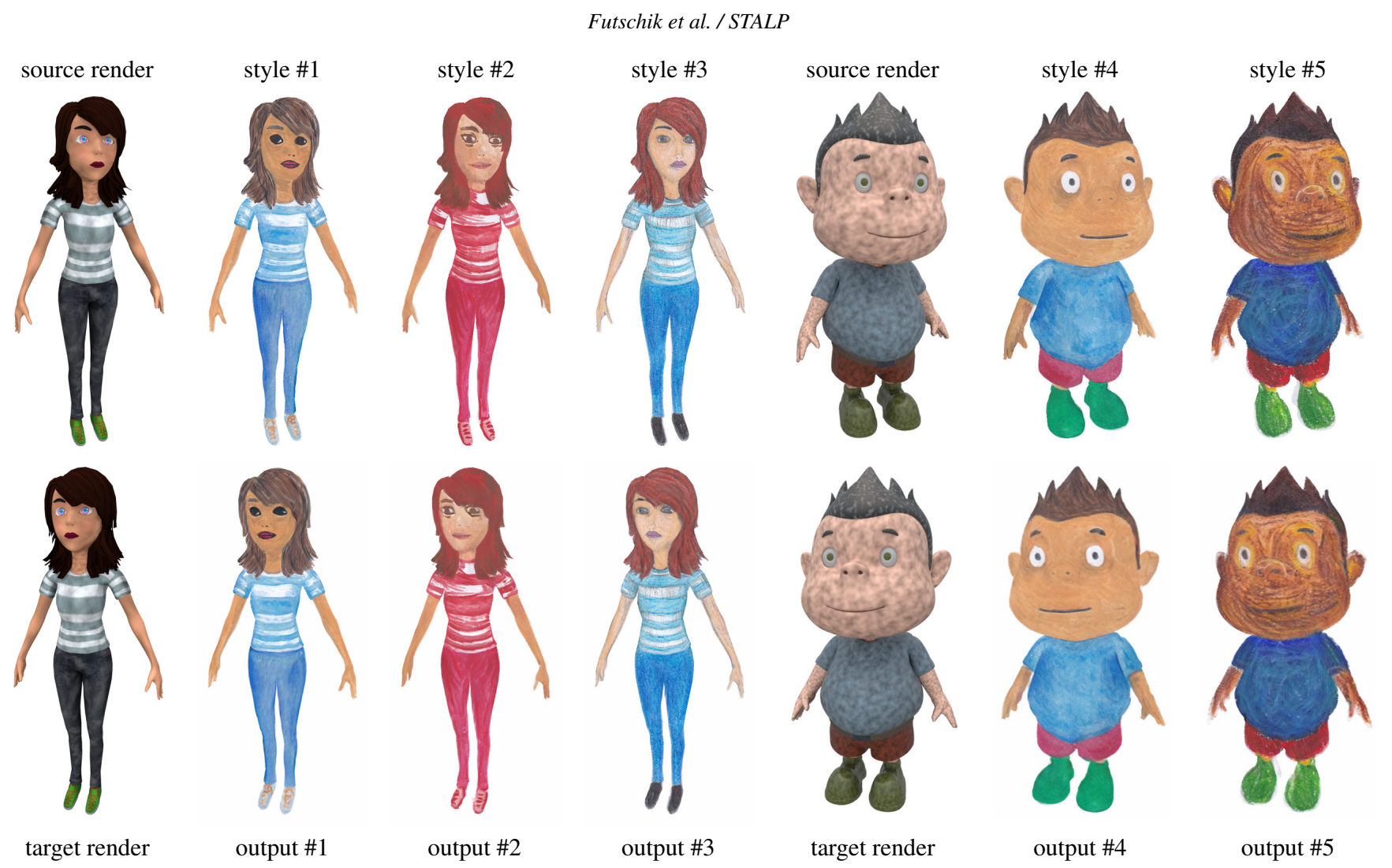

Figure 10: Stylization of 3D renders (cont.)—a colored 3D model enhanced by a noisy texture (source render) is stylized by hand using various artistic media (style \#1-\#5). The resulting image translation network $\mathcal{F}$ is then used to stylize the same 3D model (output \#1-\#5) rendered from a different viewpoint (target render) in real-time. Source styles (\#1-\#5) @ Štépánka Sýkorová, used with permission.

[SWHS97] Salisbury M. P., Wong M. T., Hughes J. F., Salesin D. H.: Orientable textures for image-based pen-and-ink illustration. In SIGGRAPH Conference Proceedings (1997), pp. 401-406.

[SZ14] Simonyan K., Zisserman A.: Very deep convolutional networks for large-scale image recognition. CoRR abs/1409.1556 (2014).

[TFF*20] TEXler O., FUTSChiK D., FiŠER J., LUKÁČ M., LU J., SHeChTMAN E., SÝKORA D.: Arbitrary style transfer using neurallyguided patch-based synthesis. Computers \& Graphics 87 (2020), 62-71.

[TFK* 20] TEXLER O., FUTSCHIK D., KuČERA M., JAMrišKa O., SOCHOROVÁ S., CHAI M., TulYakov S., SÝKORA D.: Interactive video stylization using few-shot patch-based training. ACM Transactions on Graphics 39, 4 (2020), 73.

[UVL16] Ulyanov D., Vedaldi A., Lempitsky V. S.: Instance normalization: The missing ingredient for fast stylization. CoRR $a b s / 1607.08022$ (2016).

[WLT*19] Wang T.-C., LiU M.-Y., Tao A., LiU G., Kautz J., Catanzaro B.: Few-shot video-to-video synthesis. In Advances in Neural Information Processing Systems (2019), pp. 5014-5025.

[ZPIE17] ZhU J.-Y., PARK T., Isola P., EFros A. A.: Unpaired image-to-image translation using cycle-consistent adversarial networks. In Proceedings of IEEE International Conference on Computer Vision (2017), pp. 2242-2251. 
source photo

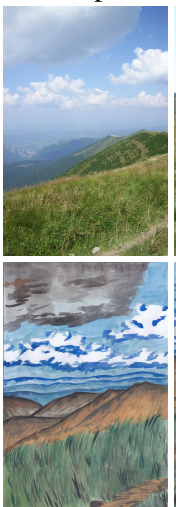

source style target panorama

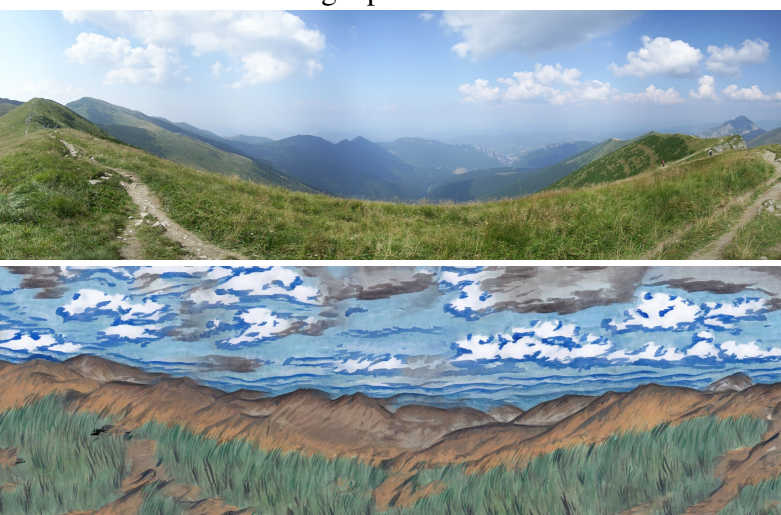

our approach
Liao et al.

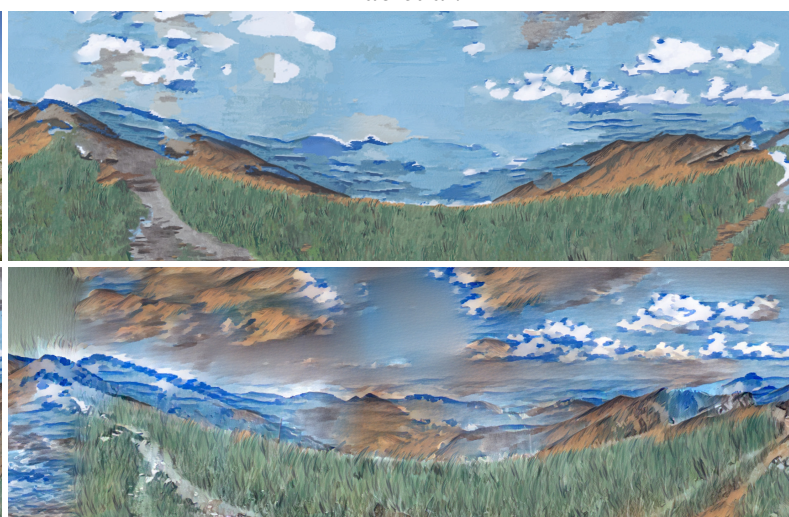

Kolkin et al.

Figure 11: Panorama stylization results-a photo (source photo) is selected from a set of shots taken around the same location by rotating a camera (target panorama) and stylized using different artistic media (source style). The network is then trained using the stylized pair and a subset of photos of the panoramic image (target panorama). Finally, the network is used to stylize each shot, and the entire panorama is stitched together (our approach). In contrast to previous techniques (Liao et al. [LYY*17] and Kolkin et al. [KSS19]) our approach better preserves essential artistic features and transfers them into appropriate semantically meaningful locations. See also results with additional styles in Fig. 12. Source style @ Štěpánka Sýkorová, used with permission.

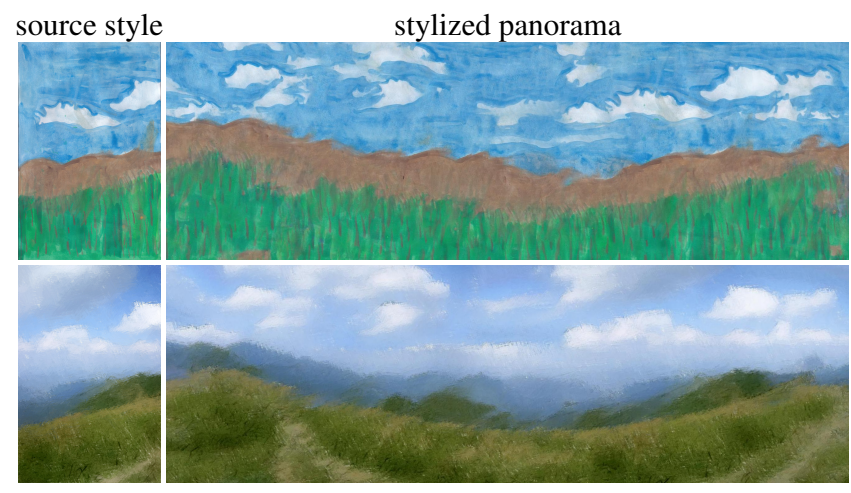

Figure 12: Panorama stylization results (cont.)—two additional artistic styles (source style) used to stylize the panorama shown in Fig. 11. Note how our approach (stylized panorama) handles also a higher level of abstraction (first row). Source style (top row) (C) Jolana Sýkorová, used with permission. 


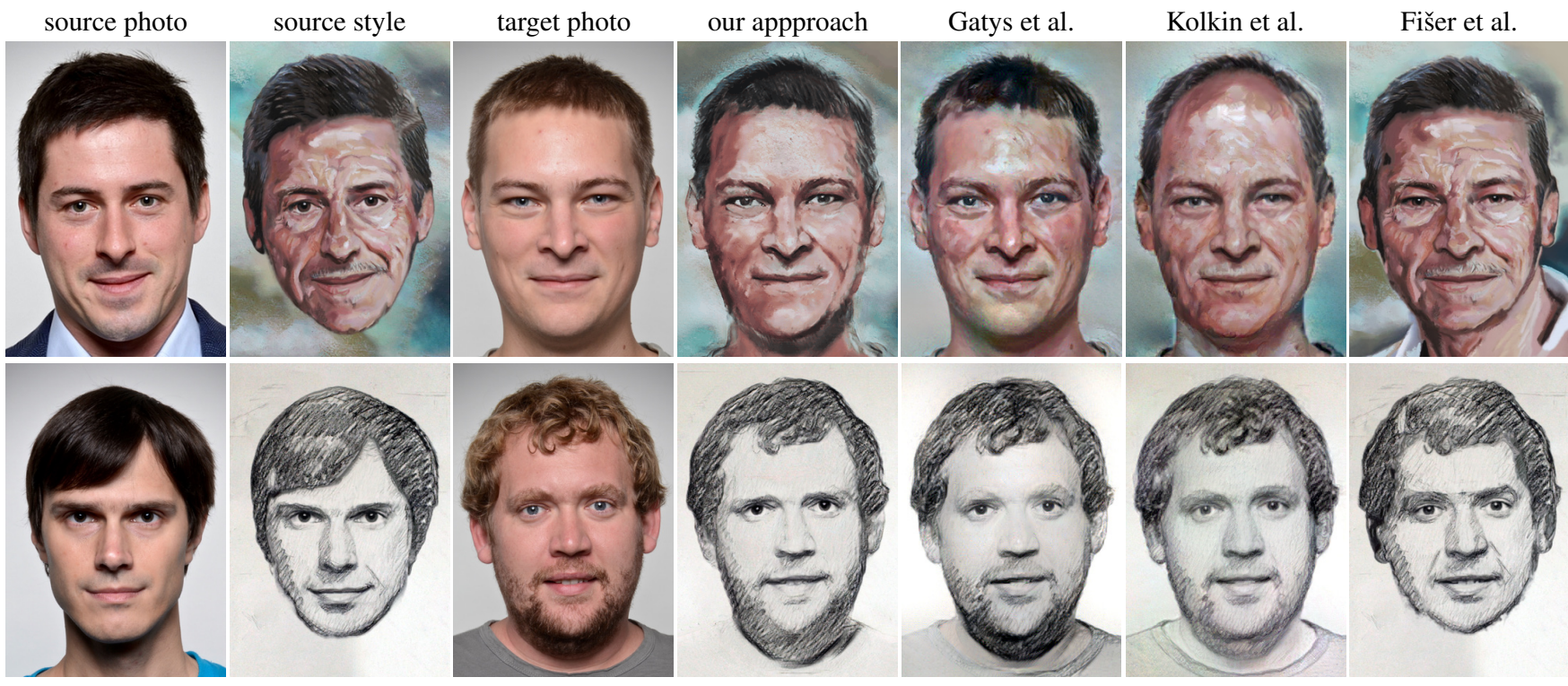

Figure 13: Stylization of portraits-a portrait photo (source photo) taken from a set of portraits captured under similar lighting conditions is stylized by an artist (source style). The network is then trained on the stylized pair and other portraits from the original set (target photo). Once trained the network can be used to stylize the other portraits (our approach). Even in this more challenging scenario our method produces a reasonable compromise between style and identity preservation whereas concurrent techniques suffer either from loosing important high-frequency details (Gatys et al. [GEB16] and Kolkin et al. [KSS19]) or have difficulties to retain identity (Fišer et al. [FJS*17]). Source style (top row) () Graciela Bombalova-Bogra and style (bottom row) () Adrian Morgan, used with permission.

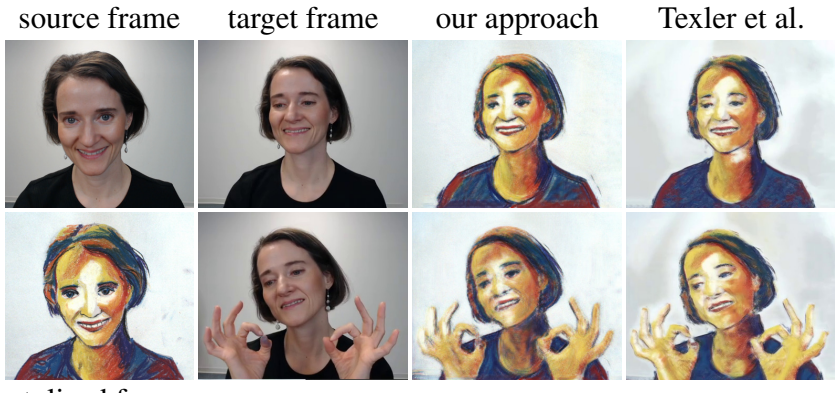

stylized frame

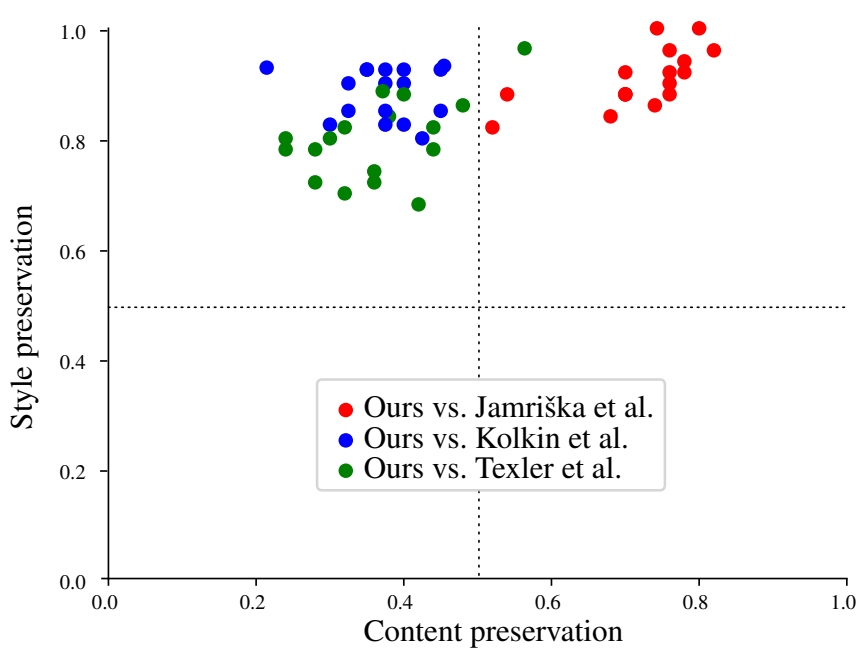

Figure 15: Results of perceptual study-each point represents aggregated votes over a group of 10 participants. On the $x$ axis we depict the percentage of answers in favor of content preservation of our method while on the y axis we show the style reproduction percentage. Comparisons were performed with the method of Jamriška et al. [JST*19] (red points), Kolkin et al. [KSS19] (blue points), and Texler et al. [TFF $\left.{ }^{*} 20\right]$ (green points). From the graph it is visible that our method is observed to reproduce style notably better than previous works. It also outperforms the method of Jamriška et al. w.r.t. the content preservation, however, Kolkin et al. as well as Texler et al. are better in content preservation. 
(a)

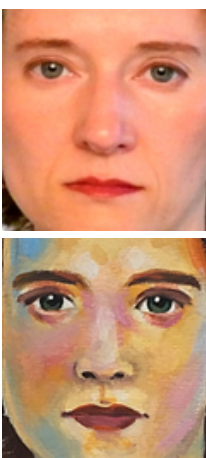

(e) (b)

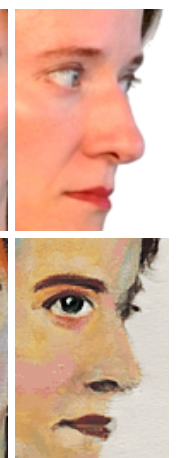

(f) (c)

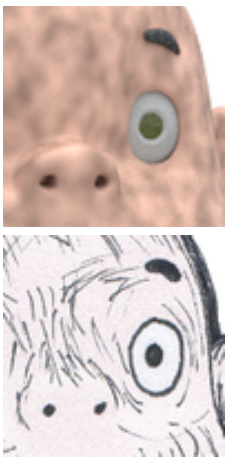

(g) (d)

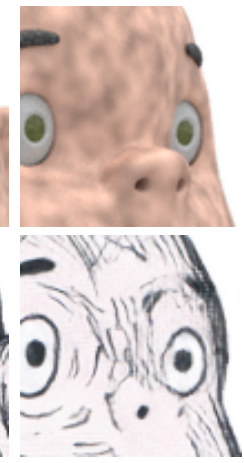

(h)

Figure 16: Limitation on generalization-although our approach usually generalizes better than concurrent stylization techniques [JST* 19, TFK 20 ], some specific features like eyes $(a, c)$ that tend to generate strong activation in selected layers of VGG network may bias the VGG loss and make the network $\mathcal{F}$ reproduce their mostly unchanged copies $(f, h)$ instead of adapting to their actual geometric distortion $(b, d)$. Video frames $(a, b)$ and style (e) @ Zuzana Studená, used with permission.

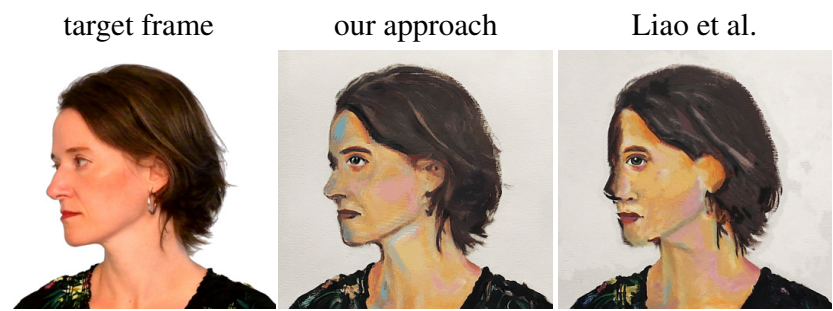

Figure 17: The advantage of using style transfer with auxiliary pairing in visual attribute transfer scenario of Deep Image Analogy [LYY*17]. Although the style's texture and semantics (see source style in Fig. 1) are preserved well in both techniques, Deep Image Analogy (Liao et al.) has difficulties in adapting to certain structural changes. Target video frame (C) Zuzana Studená, used with permission.

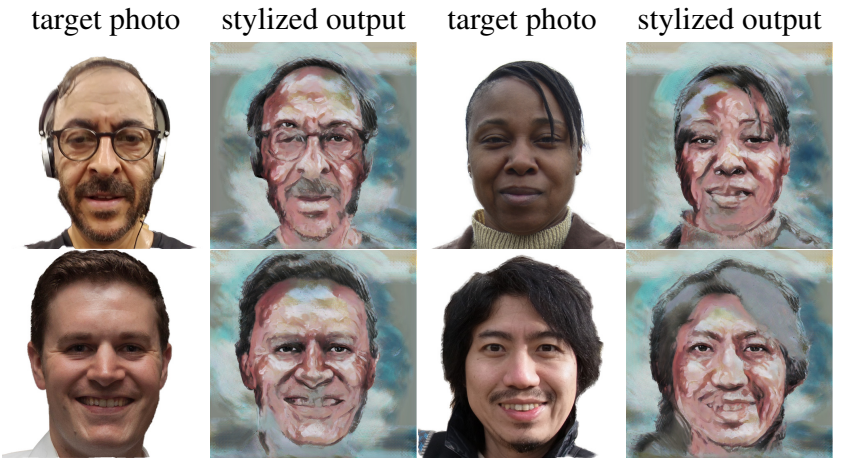

Figure 18: Limitation on a greater appearance change in the target photo-a key assumption of our method is that the domain of source and target photos is similar, e.g., photos are taken under same illumination conditions. When this requirement is not satisfied, the resulting stylization may start to show artifacts as is visible in those examples of photos taken from the FFHQ dataset [KLA19] where the illumination conditions are different to those used for the capture of source photo in Fig. 13. 